\title{
Verkehrte Welt : Der Süden finanziert den Norden
}

\section{Bruno Gurtner}

\section{(2) OpenEdition \\ 1 Journals}

Electronic version

URL: http://journals.openedition.org/sjep/372

DOI: $10.4000 /$ sjep.372

ISSN: 1663-9677

Publisher

Institut de hautes études internationales et du développement

\section{Printed version}

Date of publication: 1 novembre 2007

Number of pages: $61-84$

ISBN: 978-2-88247-069-0

ISSN: 1660-5926

Electronic reference

Bruno Gurtner, «Verkehrte Welt : Der Süden finanziert den Norden », Schweizerisches Jahrbuch für Entwicklungspolitik [Online], 26-2 | 2007, Online erschienen am: 01 Juni 2010, abgerufen am 08 September 2020. URL : http://journals.openedition.org/sjep/372 ; DOI : https://doi.org/10.4000/sjep. 372 


\title{
Verkehrte Welt: \\ Der Süden finanziert den Norden
}

\author{
Bruno Gurtner*
}

\section{Einleitung}

Viele Leute in reichen Ländern glauben, es fliesse sehr viel Geld vom Norden in die Entwicklungsländer, in Form von Entwicklungshilfe, günstigen Krediten oder privaten Investitionen. Arme Länder würden von Schuldenerlassen und von Handelserleichterungen profitieren. Doch die Leute in den reichen Ländern stellen auch fest, dass die Zahl der armen Menschen, die mit zwei oder einem Dollar oder noch weniger leben müssen, erschreckend hoch bleibt. Naheliegend ist dann der Schluss, die Hilfe sei nutzlos und bewirke nichts. Oder sie würde nur die skrupellosen und korrupten Eliten nähren.

Deshalb will der folgende Beitrag nachzeichnen, dass zwar den Entwicklungsund Transitionsländern in den letzten Jahren tatsächlich in steigendem Ausmass Kapital aus den reichen Ländern zugeflossen ist. Dieser Zustrom setzt sich nicht nur aus Hilfe, sondern umfangmässig weit stärker auch aus Krediten und Investitionen aus öffentlichen wie privaten Quellen zusammen. Doch nackte Zahlen ergeben noch kein vollständiges Bild dieser Kapitalströme. Nicht alles ist Hilfe, was als solche ausgegeben wird. Statistiken sind nicht vollständig. Insbesondere erfassen sie oft nicht alle Kapitalabflüsse.

Deshalb will dieser Beitrag den Blick vor allem auf den Kapitalabfluss wenden. Bürgerinnen und Bürger in Entwicklungsländern sehen ihr Geld aus ihren Ländern abfliessen, zum Beispiel in Form von Zins- und Amortisationszahlungen auf ihren Schulden. Durch den Abfluss massiver Gewinnrückzahlungen an transnationale Konzerne und durch Abgeltung teurer Lizenzgebühren auf Patenten, oder auch durch die Kapitalflucht ihrer eigenen Elite. Dieses Geld fehlt dann für die Entwicklung des Landes, für die Finanzierung sozialer Aufgaben.

„Und wenn Afrika ein Nettokreditor der Welt wäre?“, fragte unlängst die Zeitschrift Marchés Tropicaux \& Méditerranéens ${ }^{1}$. Für das UN Department of Economic and Social Affairs ist diese Frage bereits geklärt. Im Jahr 2006 flossen 658 Milliarden Dollar mehr vom Süden in den Norden, als umgekehrt. Die Transitionsländer verzeichneten netto einen Abfluss von 125 Milliarden Dollar ${ }^{2}$. Dieser Nettofinanztransfer, errechnet auf Grund der Zahlungsbilanzstatistiken des Internationalen Währungsfonds (IWF), sei für die Entwicklungsländer

* Ökonom, zuständig für internationale Finanzfragen bei Alliance Sud, der Arbeitsgemeinschaft Swissaid - Fastenopfer - Brot für alle - Helvetas - Caritas - HEKS. Seit Anfang 2007 präsidiert der Autor das globale Netzwerk für Steuergerechtigkeit (Tax Justice Network).

1 Dossier fiscalité, Marchés tropicaux et méditerranéens, Nr. 3201, 4. Mai 2007, <http :/www.marchestropicaux.com>.

2 United Nations, World Economic Situation and Prospects 2007 (nachstehend WESP 2007), New York, 2007, S. 58 f., <http://www.un.org/esa/policy/wess/wesp> oder <http://www.unctad.org>. 
insgesamt seit 1997 und für die Transitionsländer seit 1999 stets negativ gewesen (wir kommen später auf diesen Punkt zurück).

Im Folgenden wird analysiert, welche Arten des Kapitalflusses in welcher Weise zu diesem Mittelfluss vom Süden in den Norden beitragen, indem wir zuerst einen Blick auf die öffentliche Hilfe und die übrige öffentliche Entwicklungsfinanzierung werfen. Danach befassen wir uns mit den privaten Kapitalflüssen und anschliessend mit den Kapitalflüssen, die sich aus der Verschuldung ergeben, und ziehen eine Bilanz des Nettokapitalflusses vom Süden in den Norden. Ein spezieller Abschnitt vor dem abschliessenden Teil wird dem Thema der Kapitalflucht gewidmet. Dieser Beitrag zeichnet nach, dass umfangreiche Mittel in Form von illegalem Kapital aus den Entwicklungs- und Transitionsländern abfliessen, die in den Statistiken nicht oder nur als „Fehler und Auslassungen“ in den Zahlungsbilanzen erscheinen. Rücküberweisungen von Migrantinnen und Migranten in ihr Heimatland zwischen den einzelnen Entwicklungsländern werden in diesem Artikel nicht behandelt. Auch auf die Darstellung der Süd-SüdKapitalflüsse wird hier verzichtet.

\section{Die öffentliche Entwicklungsfinanzierung}

\section{Die bilaterale öffentliche Entwicklungshilfe}

Gemäss der Organisation für wirtschaftliche Zusammenarbeit und Entwicklung (OECD) erreichte die öffentliche Entwicklungshilfe ihrer Mitgliedsländer im Jahr 2006 insgesamt 103,9 Milliarden Dollar ${ }^{3}$. Das entspricht 0,30 Prozent des Bruttoinlandprodukts (BIP). Ein Jahr zuvor hatte sich die Hilfe noch auf 106,8 Milliarden Dollar (0,33\% des BIP) belaufen. Der Rückgang war erwartet worden und hängt mit den ausserordentlich hohen Entschuldungsmassnahmen zugunsten des Irak und Nigerias im Vorjahr zusammen ${ }^{4}$. Gemäss dem Entwicklungshilfeausschuss (DAC) der OECD ist dies aber der erste Rückgang in realen Werten seit 1997. Trotzdem ist das immer noch doppelt so viel wie im Jahr 2001 (52,5 Mrd. \$). Eine ähnliche Steigerung weist die Weltbank aus ${ }^{5}$.

Die Debatte um die Millenniumsentwicklungsziele hatte also nach Jahren stagnierender oder gar rückläufiger weltweiter öffentlicher Hilfe offensichtlich und endlich doch zu einer Trendumkehr geführt. In den 60er-Jahren hatte die öffentliche Entwicklungshilfe 0,5 Prozent des BIP erreicht; bis zur Verabschiedung der UN-Millenniumserklärung sank sie dann bis auf 0,21 Prozent. Der Monterrey-Konsensus versuchte, die zentrale Rolle der öffentlichen Entwicklungshilfe insbesondere für die ärmsten Länder wiederherzustellen und das alte 0,7-Prozent-Ziel erneut zu bekräftigen. Zahlreiche Länder bemühten sich danach um eine Erhöhung ihrer Hilfe.

3 OECD, Development aid from OECD countries fell 5.1\% in 2006, Pressemitteilung, 3. April 2007, $<$ http://www.oecd.org/dac>. Vgl. dazu auch Development Assistance Committee (DAC), Development Co-operation Report 2005, Paris, OECD, 2006.

4 Siehe unten sowie Fussnoten 12-16.

5 Gemäss Weltbank belief sich die Entwicklungshilfe (Geschenke und technische Zusammenarbeit) im Jahr 2005 auf 77,4 Milliarden Dollar. Im Jahr 2000 hatte sie noch 44 Milliarden Dollar betragen. Vg1. World Bank, Global Development Finance 2006 (nachstehend GDF 2006), Vol. 1, Tabellen 1 und A1, Washington, DC, The World Bank, 2006, <http://www.worldbank.org/gdf2006>. 
Tabelle 1: Öffentliche Entwicklungshilfe der OECD-DAC-Staaten (in Millionen Dollar zu laufenden Preisen)

\begin{tabular}{lrrrrrrr}
\hline & $\mathbf{2 0 0 0}$ & $\mathbf{2 0 0 1}$ & $\mathbf{2 0 0 2}$ & $\mathbf{2 0 0 3}$ & $\mathbf{2 0 0 4}$ & $\mathbf{2 0 0 5}$ & $\mathbf{2 0 0 6}^{\text {p }}$ \\
$\begin{array}{l}\text { Öffentliche } \\
\text { Entwicklungshilfe }\end{array}$ & 53749 & 52435 & 58292 & 69085 & 79512 & 106777 & 103940 \\
\hline- Bilaterale Hilfe & 33040 & 33522 & 39813 & 50908 & 57322 & 83109 & \\
\hline- Bilaterale Kredite & 3024 & 1602 & 939 & -1153 & -2937 & -976 & \\
\hline $\begin{array}{l}- \text { Beiträge an } \\
\text { multilaterale Institutionen }\end{array}$ & 17685 & 17311 & 17540 & 19330 & 25126 & 24644 \\
$\begin{array}{l}\text { Andere öffentliche } \\
\begin{array}{l}\text { Flüsse } \\
\hline\end{array}\end{array}$ & -4326 & -1589 & -45 & -348 & -5599 & 1430 \\
\hline
\end{tabular}

Quellen: DAC, Development Co-operation Report 2005, Paris, OECD, 2006. DAC, Pressemitteilungen

p Vorläufige Schätzung.

Doch damit ist das unmittelbare Ziel - 0,5 Prozent im Jahr 2010, 0,7 Prozent im Jahr 2015 - noch nicht erreicht. Dazu müssten die Geberstaaten ihre öffentliche Hilfe jährlich um acht Prozent steigern, eine Zuwachsrate, die deutlich über dem Wachstum der gesamten öffentlichen Ausgaben liegt ${ }^{6}$. Nur wenige Länder erfüllten bisher ein zweites Ziel, nämlich mindestens 0,2 Prozent ihres BIP den ärmsten Ländern als Hilfe zukommen zu lassen.

$\mathrm{Ob}$ Nicht-DAC-Mitglieder in genügendem Ausmass zur notwendigen Steigerung beitragen werden, bleibt abzuwarten. Immerhin haben China und Indien für die nächsten Jahre beträchtliche Zusagen gemacht. China will seine Hilfe im Rahmen seines Aktionsplans für die Zusammenarbeit mit Afrika in der Periode zwischen 2007 und 2009 verdoppeln. Ein Teil der Hilfe soll allerdings mit Darlehen und präferenziellen Exportkrediten erfolgen. Das hat bereits zu Kontroversen mit den alten Gebern geführt. Auch Mitglieder der Organisation Erdöl exportierender Länder (OPEC), die Türkei, Mexiko, Korea und ein Teil der neuen Mitglieder der Europäischen Union (EU) bekundeten ihre Absicht, die Hilfe an die ärmsten Länder zu steigern.

Der Blick auf blosse Zahlen verdeckt jedoch einige unangenehme Tatsachen. Erstens scheint die öffentliche Entwicklungshilfe gemäss José Ocampo ${ }^{7}$ im Hinblick auf die Millenniumsziele insgesamt weniger effektiv zu sein, weil sich ihre Zusammensetzung in den letzten Jahren beträchtlich verändert hat. Die Anteile für die Entschuldung, für Nothilfe und für technische Hilfe haben relativ zugenommen. Nothilfe ist nicht zur Stärkung langfristiger Entwicklung konzipiert und bestimmt. Die Entschuldungsmassnahmen brachten im Allgemeinen wenig neue Finanzmittel in die Länder, wurden oft gar zulasten der bisherigen Entwicklungshilfe finanziert (siehe unten, Schuldenströme und Entschuldung). Die technische Zusammenarbeit trage zwar in vielfältiger Weise zu positiven Entwicklungsresultaten bei, doch wie viel sie zum Kapitalzufluss beitrage, sei schwierig zu messen, befindet Richard Manning, Vorsitzender des DAC der OECD ${ }^{8}$. Insgesamt nehme die öffentliche Hilfe als Quelle der für die Regierungen der Entwicklungsländer verfügbaren Budgetmittel umfangmässig $a b$. Insbesondere die

United Nations, WESP 2007, S. 68.

José Antonio Ocampo, Jan Kregel und Stephany Griffith-Jones (eds.), International Finance and Development, London, Zed Books, 2007, S. 69.

8 Richard Manning, Statement at the Developement Committee Spring Meeting 2005, <http://go.worldbank. org/HX6ZGHS2W0>. 
ärmsten Entwicklungsländer hätten real und nach Abzug der Nothilfe und der Entschuldung weniger Hilfsgelder zur Verfügung als $1990^{9}$.

Zweitens veränderten sich gemäss Ocampo die Hilfsströme mit den wirtschaftlichen Zyklen der Geberstaaten. Die Hilfsgelder wiesen deshalb in der Vergangenheit beträchtlich höhere Schwankungen (Volatilität) auf als etwa das Bruttoinlandsprodukt oder die Fiskaleinnahmen. Sie wirkten sich prozyklisch aus, verstärkten also einerseits einen Wirtschaftsaufschwung, aber eben auch eine Wirtschaftskrise. Die Volatilität der Entwicklungshilfe hat insgesamt einen ähnlichen Effekt wie schwankende Rohstoffpreise. Sinkende öffentliche Hilfe führt in der Regel zu höheren Steuern und Ausgabenkürzungen, also zu kostspieligen fiskalischen Anpassungsprogrammen. Die ärmsten Länder, die stark von öffentlicher Entwicklungshilfe abhängen, sind demzufolge nicht weniger von Schwankungen im Kapitalzufluss betroffen als die schon etwas fortgeschritteneren Länder, die eher vom Zufluss privater Kapitalflüsse abhängen ${ }^{10}$. Unstetig zufliessende Hilfsgelder vermindern die Voraussehbarkeit der Hilfe und beeinträchtigen die mittelfristige Planungsfähigkeit der Entwicklungsländer. Erschwerend wirkt sich auch aus, dass die Zusagen der Geber und ihre effektiven Auszahlungen häufig weit auseinanderklaffen.

Drittens ist die öffentliche Entwicklungshilfe sehr oft mit strengen wirtschaftspolitischen Bedingungen (Privatisierung, Liberalisierung, Ausgabenkürzungen usw.) verknüpft. Eigentlich ist heute die Erkenntnis Allgemeingut, dass die Geber ihre Ziele mit Konditionalität nur schlecht erreichen können. Reformpolitiken sind dann erfolgreich, wenn sie im betreffenden Land breit mitgetragen werden. Dennoch wird die Hilfe in vielen Ländern mit Vorschriften und Einschränkungen verbunden, welche den politischen Entscheidungsspielraum (policy space) und damit die Demokratie der Entwicklungsländer einschränken. Die Hilfe hat oft mehr mit den Interessen der Geber, denn mit jenen der Begünstigten zu tun.

Viertens konzentrieren die bilateralen Geber ihre Hilfe auf relativ wenige Länder. Die zwanzig grössten Empfängerländer erhielten im Jahr 2004 mehr als die Hälfte der bilateralen Hilfsgelder (1980 waren es allerdings noch fast zwei Drittel gewesen). Und weniger als die Hälfte der Entwicklungsländer erhalten 90 Prozent der Hilfe der DAC-Länder (vgl. Grafik 1). Das sei dem Herdenverhalten privater Investoren nicht unähnlich, monieren Ocampo und MitautorInnen ${ }^{11}$.

Diese und ähnliche Tatsachen bewegten Action Aid International im Jahr 2005 in einer umfangreichen Studie zur Aussage, dass mehr als die Hälfte der offiziellen bilateralen Hilfe so genannte „Phantomhilfe“ sei, Hilfe, die nicht einem realen Ressourcentransfer für die Armutsbekämpfung entspreche ${ }^{12}$. In einer zweiten Studie, die iim Juli 2006 publiziert wurde, doppelte Action Aid International nach und nahm vor allem die technische Hilfe der reichen Länder unter die Lupe. Der Einsatz von teuren Konsulenten sowie Forschung und Ausbildung würden zu oft die Interessen der Geber bevorzugen und zu unangepassten Lösungen nördlichen

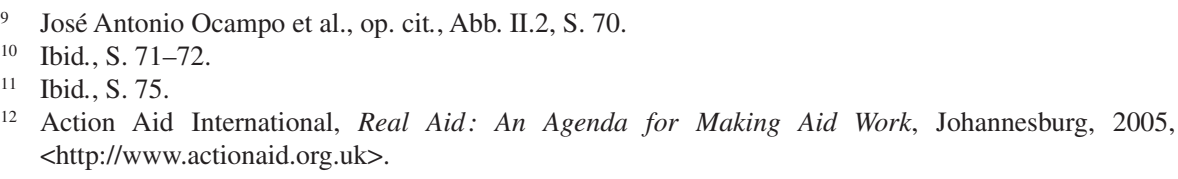


Ursprungs führen ${ }^{13}$. Von insgesamt 79 Milliarden Dollar der öffentlichen Hilfe von DAC-Mitgliedern im Jahr 2004 seien wiederum 37 Milliarden „Phantomhilfe": 7 Milliarden Dollar seien nicht für die Armutsbekämpfung bestimmt gewesen, rund 6 Milliarden seien als Entschuldung doppelt gezählt, 12 Milliarden als preislich überbewertete und ineffiziente technische Hilfe. 2,5 Milliarden Dollar (gerundeter Betrag) seien durch Lieferbindungen (tied aid) und 8 Milliarden durch mangelnde Koordination unter den Gebern verloren gegangen. 2 Milliarden seien als Migrationsauslagen in den Geberländern selber ausbezahlt worden. Exzessive administrative Kosten hätten 70 Millionen Dollar betragen.

\section{Grafik 1: Konzentration der öffentlichen Entwicklungshilfe in den Empfängerländern, 1980-2004}

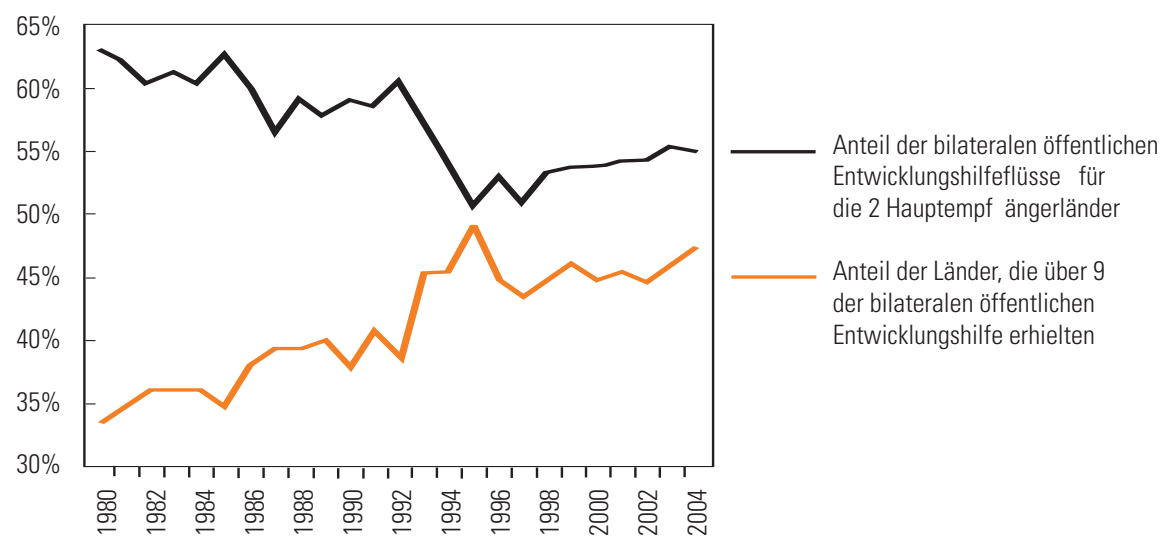

Quelle: José Antonio Ocampo, Jan Kregel und Stephany Griffith-Jones (eds.), International Finance and Development, London, Zed Books, 2007, S. 75.

Ein ähnliches Bild zeichnet eine im Frühjahr 2006 erschienene gemeinsame Studie zahlreicher europäischer NRO zur Hilfe der EU-Länder. Danach entsprachen 13,5 Milliarden Euro oder rund ein Drittel der öffentlichen Hilfe der EU-Länder gegenüber den Entwicklungsländern des Jahres 2005 keinem realen Hilfsstrom ${ }^{14}$. Der DAC zählte Entschuldungsmassnahmen in Höhe von 11,8 Milliarden Euro als Hilfe, davon allein 9,2 Milliarden für den Irak und Nigeria. 840 Millionen Euro als Ausgaben für Flüchtlinge in den EU-Ländern berechnete die OECD ebenso als Hilfe wie 910 Millionen Euro als Ausgaben für Studierende aus Entwicklungsländern in den EU-Ländern. Die entsprechenden Zahlen für die einzelnen Länder fallen sehr unterschiedlich aus ${ }^{15}$.

13 Action Aid International, Real Aid 2: Making Technical Assistance Work, Johannesburg, 2006, $<\mathrm{http}$ ://www.actionaid.org.uk>.

14 Eurodad (ed.), EU Aid: Genuine Leadership or Misleading Figures? An Independent Analysis of European Aid Figures, Joint European NGO Report, April 2006, <http://www.eurodad.org> oder $<$ http://www.concordeurope.org $>$.

15 In der Schweiz wies der gemeinsame Jahresbericht von DEZA und SECO für das Jahr 2005 die gemäss DAC als Hilfe angerechneten Ausgaben für Asyl und Rückkehr mit 8 Prozent aus (Vorjahr $14 \%$ ), während die Entschuldungsmassnahmen 13 Prozent betrugen (1\%). Vgl. dazu DEZA und SECO, Jahresbericht der internationalen Zusammenarbeit der Schweiz, diverse Jahrgänge, Tabelle 1, $<$ http://www.ddc.admin.ch>. 
Eurodad und Concord bestätigen dieses Bild anhand der OECD-Zahlen für das Jahr $2006^{16}$.

Fazit: Die öffentliche Entwicklungshilfe ist in den letzten Jahren gestiegen, hat aber nach wie vor noch nicht den dringend nötigen Umfang erreicht, damit die Millenniumsentwicklungsziele finanziert werden können. Das Volumen ist aber auch inflationär aufgebläht. Ein beträchtlicher Teil der statistisch als Entwicklungshilfe ausgewiesenen Summe entspricht nicht einem realen Zufluss an Mitteln in die Entwicklungsländer. Asylkosten, Drogen- und Terrorbekämpfung, Massnahmen, die in erster Linie der Sicherheit der Industrieländer dienen, sind keine Entwicklungshilfe. Ein anderer Teil - wie Entschuldung, technische Hilfe, gebundene Hilfe - ist in den Statistiken überbewertet.

\section{Andere öffentliche Entwicklungsfinanzierung}

Neben der öffentlichen Entwicklungshilfe werden auch andere Finanzströme aus bilateralen und multilateralen Quellen zur öffentlichen Entwicklungsfinanzierung ${ }^{17}$ gezählt. $\mathrm{Zu}$ den bilateralen öffentlichen Leistungen (exklusive der Hilfe) zählen insbesondere bilaterale Kredite und öffentlich garantierte Exportkredite. Nach Angaben der Weltbank sind diese Leistungen in den letzten Jahren netto rückläufig, insbesondere weil grosse Schuldnerländer wie Russland, Polen, Peru und andere ihre Schulden gegenüber den Ländern des Pariser Klubs zurückbezahlt haben. Für das Jahr 2005 beziffert die Weltbank den Rückgang auf 27 Milliarden Dollar ${ }^{18}$.

Die bilateralen Geber haben zudem in den letzten Jahren ihre direkte Kredittätigkeit gegenüber Entwicklungsländern im Allgemeinen zurückgefahren und sind dazu übergegangen, ihre Leistungen in Geschenkform zu erbringen. Direkte bilaterale Kredite der Industrieländer haben deshalb relativ an Bedeutung verloren.

Zur öffentlichen Entwicklungsfinanzierung gehören aber auch die Aktivitäten der multilateralen Finanzierungsinstitute (IFIs). Grösstenteils haben sie in der Vergangenheit ihre Mittel in Form von Darlehen gewährt. Länder mit mittlerem Einkommen zahlen einen annähernd marktüblichen Zins. Die IFIs nehmen dank ihrer Kapitalbasis (die Einlagen der Mitgliederstaaten) und ihrem guten Schuldnerstatus auf den privaten Kapitalmärkten Kredite zu relativ günstigen Zinsbedingungen auf, die sie dann ihrerseits den Entwicklungsländern mit einem geringen Zinsaufschlag als Kredite weitergeben. Die Entwicklungsländer kamen

16 Concord und Eurodad, Hold the Applause! EU Governments Risk Breaking Aid Promises, Brüssel, April 2007, <http://www.concordeurope.org> und <http://www.eurodad.org>. Vgl. auch OECD, Development Aid from OECD Countries fell 5.1\% in 2006, op. cit. Die Schweizer Hilfe fiel um 9\%, insbesondere, weil die Aufwendungen für die Restrukturierung öffentlicher Schulden (Irak, Nigeria) um 156 Mio. Fr. geringer ausfielen als im Jahr zuvor. Damit beträgt der Anteil am BIP noch 0,39\% oder nur noch 0,30\%, wenn die Ausgaben für Asylsuchende, die Stipendien für Studierende aus Entwicklungsländern in der Schweiz und die Entschuldungsmassnahmen nicht eingerechnet werden.

17 Der Entwicklungshilfeausschuss der OECD (DAC) unterscheidet zwischen der öffentlichen Entwicklungshilfe zu konzessionellen Bedingungen (ODA) und der öffentlichen Entwicklungsfinanzierung (ODF). Zu dieser gehören vor allem konzessionelle und nichtkonzessionelle multilaterale Leistungen sowie bilaterale Leistungen mit keinem oder einem geringen konzessionellen Anteil. Schliesslich führt er noch weitere öffentliche Finanzströme auf (OOF), welche nicht Hilfe als Zweck aufweisen.

18 World Bank, GDF 2006, Vol. 1, S. 7. Zum Pariser Klub siehe <http://www.clubdeparis.org>. 
so in den Genuss von Mitteln, die in der Regel billiger waren, als wenn sie selber an den privaten Kapitalmärkten Gelder aufgenommen hätten.

Den ärmsten Ländern vergeben die IFIs mit ihren Entwicklungsfonds (den weichen Kreditlinien) langfristige, zinslose Darlehen mit einer minimalen Kommission. Diese Entwicklungsfonds müssen periodisch durch neue Beitragszahlungen der Geberländer aufgestockt werden und werden teilweise aus anderen Quellen finanziert. So fliessen Rückzahlungen der Darlehensnehmer wiederum in diese Entwicklungsfonds zurück. Zudem finanzieren die Entwicklungsbanken ihre ,weichen“ Kreditlinien mit den Gewinnen, die sie mit der Kreditvergabe zu kommerziellen Bedingungen an die Länder mit mittlerem Einkommen erzielen. In neuerer Zeit sind diese Fonds vermehrt dazu übergegangen, ihre Mittel statt als Darlehen in Geschenkform zu vergeben, was zu einem höheren Refinanzierungsdruck führt. Bekanntester Entwicklungsfonds ist die Weltbank-Tochter IDA, deren 15. Wiederauffüllung im Laufe des Jahres 2007 ausgehandelt wird ${ }^{19}$.

Über die Rolle und die Aufgaben dieser IFIs und ihrer Entwicklungsfonds sowie deren Anpassung an die neuen Gegebenheiten ist in den letzten Jahren sehr intensiv diskutiert worden. Von Interesse ist hier die Frage, wie viel die IFIs zum Mittelfluss beigetragen haben.

Die Weltbank (IBRD) als grösste multilaterale Entwicklungsbank verzeichnete mit ihren Darlehen zu kommerziellen Bedingungen letztmals im Jahr 2001 einen positiven Nettoressourcenfluss. Seither haben die Entwicklungsländer mehr Rückzahlungen geleistet, als sie neue Kredite erhielten. Von 2002 bis 2006 sind insgesamt 24 Milliarden Dollar aus Entwicklungsländern an die Bank zurückgeflossen. Bei der IDA waren die Auszahlungen von zinslosen Darlehen und von Geschenken an die ärmsten Entwicklungsländer bisher stets höher als die Rückzahlungen. Insgesamt hatte die IDA Ende 2006 ausstehende Kredite im Ausmass von 127 Milliarden Dollar ${ }^{20}$.

Auch die Interamerikanische Entwicklungsbank (IDB) wies im letzten Geschäftsjahr netto mehr Rückzahlungen auf, als sie neue Kredite gewährte ${ }^{21}$. Dasselbe erwartet in Zukunft die Asiatische Entwicklungsbank (AsDB), und sie ist deshalb auf der Suche nach einem neuen Rollenverständnis. Die Afrikanische Entwicklungsbank (AfDB) konnte hingegen ihre Ausleihungen in den letzten Jahren stetig steigern.

Insgesamt haben die Entwicklungsländer in den letzten fünf Jahren in Form von Amortisations- und Zinszahlungen stets mehr Geld an die multilateralen Geber bezahlt, als sie von ihnen neue Kredite erhielten (vgl. Tabelle 2) ${ }^{22}$. Auszahlungen der IFIs zu konzessionellen Bedingungen für die ärmsten Entwicklungsländer blieben insgesamt jedoch stets höher als die Rückzahlungen.

19 Vgl. dazu <http://www.worldbank.org/ida $>$.

20 World Bank, Annual Report, div. Jg., <http://www.worldbank.org/annualreport>.

${ }^{21}$ Inter-American Development Bank (IDB), Annual Report 2006, Washington, DC, 2007, $<$ http://www.iadb.org/exr/ar2006>.

22 World Bank, GDF 2006, Vol. 2, S. 3. 
Tabelle 2: Kapitalflüsse der multilateralen Entwicklungsagenturen, 2000-2005 (in Milliarden Dollar)

\begin{tabular}{lrrrrr}
\hline & $\mathbf{2 0 0 0}$ & $\mathbf{2 0 0 2}$ & $\mathbf{2 0 0 3}$ & $\mathbf{2 0 0 4}$ & $\mathbf{2 0 0 5}$ \\
\hline Kapitalauszahlungen total & 35.1 & 32.3 & 37.9 & 33.9 & 31.4 \\
\hline Amortisationszahlungen total & 23.2 & 30.9 & 36.5 & 31.4 & 29.2 \\
\hline Zinszahlungen total & 15.6 & 13.7 & 12.3 & 11.1 & 11.1 \\
\hline Nettotransfer total & -3.7 & -12.3 & -10.9 & -8.6 & -8.9 \\
\hline Kapitalauszahlungen konzessionell & 8.2 & 9.9 & 10.5 & 12.0 & 10.8 \\
\hline Amortisationen konzessionell & 2.5 & 2.5 & 2.8 & 3.3 & 3.7 \\
\hline Zinszahlungen konzessionell & 1.2 & 1.3 & 1.4 & 1.7 & 1.8 \\
\hline Nettotransfer konzessionell & +4.5 & +6.1 & +6.3 & +7.0 & +5.3 \\
\hline
\end{tabular}

Quelle: World Bank, Global Development Finance 2006, Vol. 2, Washington, DC, The World Bank, 2006.

Nicht in diesen Zahlen eingeschlossen sind die Leistungen des IWF. Theoretisch erfüllt der IWF nicht die Funktion einer Entwicklungsbank. Er soll kurzfristige Überbrückungskredite in Finanz- und Zahlungsbilanzkrisen leisten. Weil aber sehr viele Entwicklungsländer Überbrückungsabkommen mehrfach ohne Unterbruch erneuern mussten, wurde der IWF faktisch doch zu einer Art Agentur für Entwicklungsfinanzierung, insbesondere bei den einkommensschwachen Ländern, denen die Mittel ebenfalls zu sehr günstigen finanziellen Bedingungen zuflossen. Die Kredite des IWF und die Rückzahlungen an den IWF verlaufen sehr zyklisch. In der Folge von Finanz- und Zahlungsbilanzkrisen (wie beispielsweise bei der Finanzkrise asiatischer Länder) steigen die Auszahlungen jeweils stark an. Die Rückzahlungsfristen sind deutlich kürzer als beispielsweise bei der Weltbank (mit Ausnahme der verbilligten Leistungen an die ärmsten Länder). Demzufolge steigen die Rückzahlungen dann jeweils nach einer Karenzfrist in der Nachkrisenzeit wiederum stark an. So auch jetzt wieder. In den letzten vier Jahren zahlten die Schuldnerländer massiv ausstehende Kredite zu einem guten Teil vorzeitig zurück. Neue Kreditauszahlungen blieben sehr gering, so dass im Geschäftsjahr 2006 netto 34,3 Milliarden Dollar zurückflossen. Weil der IWF sein internes Budget mit Kommissionen auf den Ausleihungen finanziert, geriet er in eine Finanzklemme ${ }^{23}$.

Fazit: Die Gründe für die rückläufige Inanspruchnahme von Krediten bei den multilateralen Institutionen sind vielfältig. Beim IWF führt das Ausbleiben von Finanzkrisen zu einem Rückgang in der Nachfrage nach seinen Überbrückungskrediten. Von nicht unerheblicher Bedeutung ist allerdings auch, dass sich etliche Länder von den wirtschaftspolitischen Auflagen des IWF lossagen wollten. Die Massnahmen des IWF zur Abwehr von Finanzkrisen wurden von vielen Ländern als unangepasst und krisenverstärkend beurteilt.

Für die geringere Inanspruchnahme von Krediten der Entwicklungsbanken hauptsächlich durch die Länder mit mittlerem Einkommen - werden in der entwicklungspolitischen Diskussion andere Argumente angeführt. Der weltweit gute Wirtschaftsverlauf, hohe Exporterlöse, Schuldenrückzahlungen und die gesteigerte Kreditfähigkeit vieler Länder sowie die sehr liquiden internationalen Kapitalmärkte mit relativ tiefen Risikozuschlägen haben den Schwellenländern und etlichen Ländern mit mittlerem Einkommen Alternativen ermöglicht. In

23 IMF, Annual Report, div. Jg., <http://www.imf.org/external/pubs/ft/ar>. Vgl. dazu auch Bruno Gurtner, „Dem IWF laufen die Kunden davon. Identitätskrise und akute Finanzprobleme“, Global+, Nr. 21, Frühling 2006. 
zahlreichen Ländern sind eigene Kapitalmärkte entstanden, so dass die externe Kreditaufnahme durch eine inländische Verschuldung ersetzt werden konnte. Auch bei der Weltbank scheint eine Rolle zu spielen, dass strukturelle Reformen, Sozial- und Umweltstandards als zu hart erachtet werden.

\section{Private Kapitalflüsse}

Die privaten Kapitalflüsse in Entwicklungs- und Transitionsländer haben markant zugenommen, und gleichzeitig hat ihre Zusammensetzung beträchtliche Änderungen erfahren. Das ist weitgehend eine Folge der von den BrettonWoods-Institutionen (Internationaler Währungsfonds und Weltbank), den Industrieländern und den internationalen Finanzunternehmen seit den 70er-Jahren vorangetriebenen Kapitalverkehrsliberalisierung sowie natürlich auch des technischen Fortschritts im Kommunikationswesen.

Üblicherweise wird zwischen privaten Investitionsflüssen und schuldenwirksamen privaten Kapitalflüssen unterschieden. $\mathrm{Zu}$ den Ersteren gehören direkte Auslandsinvestitionen (Foreign Direct Investment, FDI) und Investitionen in die wachsenden Wertschriftenbörsen der Schwellenländer (Portfolioinvestitionen). Schuldenwirksame Kapitalflüsse sind Obligationen- und Grosskreditaufnahmen. Private Kapitalflüsse erreichen mittlerweile ein Vielfaches der öffentlichen Entwicklungsfinanzierung.

In den vergangenen gut dreissig Jahren waren drei Wellen in der Entwicklung der privaten Kapitalflüsse zu verzeichnen. Der erste Boom war hauptsächlich vom Recycling der Überschüsse der Erdöl produzierenden Länder getrieben und führte zu einem raschen und massiven Anstieg von Bankausleihen an Entwicklungsländer. Dem folgte in den 70er-Jahren die durch die US-amerikanische Zinssteigerungspolitik ausgelöste Schuldenkrise. In den 90er-Jahren trugen stark wachsende FDI und Portfolioinvestitionen zum zweiten Boom bei, der mit der Finanzkrise einiger asiatischer Länder, Russlands, Argentiniens und Brasiliens endete.

$\mathrm{Zu}$ Anfang der laufenden Dekade sanken die privaten Kapitalflüsse zuerst. Ab 2003 haben sich die privaten Kapitalflüsse in einer dritten Welle wieder erholt und erreichten gemäss Weltbank-Statistiken mit rund 490 Milliarden Dollar einen neuen Höhepunkt ${ }^{24}$ (vgl. Tabelle 3 ).

Tabelle 3: Private Kapitalflüsse, 2000-2005 (in Milliarden Dollar)

\begin{tabular}{lrrrrrr}
\hline & $\mathbf{2 0 0 0}$ & $\mathbf{2 0 0 1}$ & $\mathbf{2 0 0 2}$ & $\mathbf{2 0 0 3}$ & $\mathbf{2 0 0 4}$ & $\mathbf{2 0 0 5}$ \\
\hline Private Flüsse & 187.6 & 154.4 & 171.5 & 271.9 & 396.6 & $\mathbf{4 9 0 . 5}$ \\
\hline Private Investitionsflüsse & 182.9 & 183.3 & 166.1 & 186.8 & 248.8 & 298.9 \\
\hline Direkte Auslandsinvestitionen & 168.8 & 176.9 & 160.3 & 161.6 & 211.5 & 237.5 \\
\hline Portfolioinvestitionen & 14.1 & 6.4 & 5.8 & 25.2 & 37.3 & 61.4 \\
\hline Private Kreditflüsse & 4.7 & -28.9 & 5.5 & 85.1 & 147.8 & 191.6 \\
\hline Obligationen (Bonds) & 20.5 & 11.0 & 10.8 & 26.4 & 43.0 & 61.7 \\
\hline Bankkredite & -5.2 & -10.8 & -2.8 & 9.8 & 39.4 & 67.4 \\
\hline Andere Kredite & -3.8 & -6.3 & -6.8 & -5.9 & -4.6 & -6.7 \\
\hline Kurzfristige Kredite & -6.8 & -22.7 & 4.2 & 54.9 & 70.0 & 69.3 \\
\hline
\end{tabular}

Quelle: World Bank, Global Development Finance 2006, Vol. 1, Washington, DC, The World Bank, 2006, Tabellen 1 und A.1.

p Provisorische Schätzung.

24 World Bank, GDF 2006, Vol. 1, Tabellen 1 und A1. 


\section{Private Investitionsflüsse}

Die privaten Investitionsflüsse haben sich seit dem Jahr 2002 nahezu verdoppelt und erreichten Ende 2005 fast 300 Milliarden Dollar. Die direkten Auslandsinvestitionen (FDI) tragen den grössten Teil zu dieser Steigerung bei. Der Zustrom verminderte sich zu Beginn des laufenden Jahrzehnts relativ gering, stieg aber seit 2002 um ein Drittel. Portfolioinvestitionen haben sich in diesem Zeitraum verzehnfacht. Die UN-Konferenz für Handel und Entwicklung (UNCTAD) erwartet bei den FDI für das Jahr 2006 eine Steigerung des Zustroms von 10 Prozent für alle Entwicklungsländer, für die Transitionsländer sogar einen Anstieg von 56 Prozent ${ }^{25}$.

Eine Reihe von Organisationen publizieren Daten über internationale Investitionsströme, so die Weltbank, die UNCTAD und das den internationalen Grossbanken nahestehende Institute of International Finance (IIF) in Washington ${ }^{26}$. Ihre Statistiken unterscheiden sich durch ihre unterschiedlichen Erfassungsquellen und -definitionen. Sie umfassen allerdings nicht sämtliche mit Investitionen zusammenhängenden Transaktionen. Sie erfassen Neuinvestitionen. Das können von aussen zuströmende Gelder sein, reinvestierte Gewinne früherer Investitionen oder Investitionen, die mittels konzerninterner Kredite oder mit Kreditaufnahmen an lokalen Märkten finanziert werden. Abgezogen werden Rückzüge früher getätigter Investitionen (Desinvestitionen).

Nicht berücksichtigt in diesen Statistiken über Finanzflüsse sind Gewinnrückführungen aller Art wie Dividenden, Zinsen, Royalties, Lizenzen. Diese Zahlen werden also zu Unrecht als Nettoströme (Neuinvestitionen minus Desinvestitionen) bezeichnet. Derselbe Vorbehalt ist bei den Portfolioinvestitionen anzubringen $^{27}$. Wird hingegen ein Aufkauf einer lokalen Unternehmung durch einen ausländischen Konzern mittels Kreditaufnahme am lokalen Markt finanziert, so erfolgt in Wirklichkeit kein Zustrom von Kapital. Die Statistiken über die Finanzflüsse betrachten diese Operation dennoch als einen Kapitalzufluss.

Private Investitionsflüsse konzentrieren sich nach wie vor auf einige wenige Länder und bestimmte Wirtschaftssektoren. Die zehn grössten Schwellenlän$\operatorname{der}^{28}$ ziehen allein zwei Drittel der FDI an. In die ärmsten Länder flossen lediglich 23 Milliarden direkte Auslandsinvestitionen, davon nach Afrika südlich der Sahara lediglich 18 Milliarden, vorzugsweise in die Erdöl- und Bergbauindustrie. Die Statistiken der UNCTAD belegen, dass zumindest in einigen Ländern FDI auch in die Landwirtschaft, in Brauereien und in die Leichtindustrie flossen $^{29}$.

Die verschiedenen Komponenten der FDI sowie die Portfolioinvestitionen haben selbstverständlich unterschiedliche Auswirkungen auf die Wirtschaft der

25 UNCTAD, Foreign Direct Investment Rose by $34 \%$ in 2006, Pressemitteilung, 9. Januar 2007, $<$ http://www.unctad.org/press >.

26 World Bank, GDF 2006. UNCTAD, World Investment Report (nachstehend WIR), <http://www. unctad.org>. Institute of International Finance, Capital Flows to Emerging Market Countries, $<$ http://www.iif.com>.

27 Diese Rückführungen werden hingegen in den Zahlungsbilanzstatistiken innerhalb der Bilanz der laufenden Zahlungen (Current Account Balance) erfasst. Wir kommen weiter unten darauf zurück.

28 China, Russland, Brasilien, Mexiko, Tschechien, Polen, Chile, Südafrika, Indien und Malaysia.

29 UNCTAD, WIR 2004. 
Entwicklungsländer (Rückbindung an die lokale Wirtschaft, Spill-over, Arbeitsplätze, Wissenstransfer, Umwelt, Wettbewerb, Sektorpolitik etc. ${ }^{30}$. Hier interessieren insbesondere die finanziellen Auswirkungen.

FDI erwiesen sich während der Finanzkrisen insgesamt als relativ stabil. Krisenländer mussten allerdings teilweise beträchtliche Abschwächungen im Zufluss hinnehmen. Am stabilsten sind Investitionen in neue Anlagen, die auf Grund strategischer Entscheidungen von transnational tätigen Unternehmen mit neuen, zusätzlichen Mitteln von aussen getätigt werden (sog. Greenfield Investments). Für die Periode zwischen 1990 und 2002 betrug ihr Anteil gemäss Weltbank insgesamt knapp zwei Drittel aller FDI, schwankt aber je nach Wirtschaftssektor. Investitionen, die mit reinvestierten, nicht repatriierten Gewinnen oder mit konzernintern gewährten Darlehen finanziert werden, schwanken hingegen deutlich stärker. Auch die wirtschaftliche Lage im Gastland kann zu beträchtlichen Schwankungen im Investitionsvolumen führen. Während der letzten Finanzkrise in Asien und Lateinamerika repatriierten die Konzerne in grossem Ausmass reinvestierte Gewinne und konzernintern gewährte Darlehen $^{31}$. Konzerne können auch zu kurzfristig grösserer Repatriierung von Gewinnen und Darlehen schreiten, wenn es ihre konzerneigene finanzielle Situation gebietet, oder wenn es wegen der wirtschaftlichen Situation des Ursprungslandes oder der Weltwirtschaft angezeigt erscheint ${ }^{32}$.

Die UNCTAD stellte für ausgewählte afrikanische Länder den FDI-Zuflüssen die Gewinnrückführungen gegenüber. Danach sind zwischen 1995 und 2003 zumindest in Angola, Botswana, der Demokratischen Republik Kongo, in Gabun, Guinea, Mali und Nigeria die Gewinnrücküberweisungen grösser als die Investitionszuflüsse gewesen ${ }^{33}$. Gewinnrückführungen können durchaus auch auf nichtsaubere Art erfolgen, z.B. mittels nicht marktüblicher Verrechnungspreise (Transfer Pricing) und mit kreativer Buchführung (siehe unten den Abschnitt über Kapitalflucht).

Transnationale Konzerne, welche in die Produktion von exportierbaren Gütern (tradable goods) investieren, scheinen weniger zu raschen und kurzfristigen Repatriierungen zu neigen als jene, die für lokale Märkte produzieren ${ }^{34}$. Letztere neigen ebenfalls mehr dazu, ihre kurzfristigen, dem Währungsrisiko ausgesetzten FDI-Anteile mit derivativen Instrumenten gegen Wechselkursschwankungen und andere Risiken abzusichern. Das kann zumindest zu temporären, aber

30 Vgl. dazu etwa Kavaljit Singh, Why Investment Matters: The Political Economy of International Investments, London; Brüssel, FERN; Sturminster Newton (UK), The Corner House; Rom, Campagna per la riforma della Banca Mondiale (CRBM) ; Delhi, Madhyam Books, 2007, <http:// www.fern.org > oder <http://www.thecornerhouse.org.uk>. Philipp Hersel und Sebastian von Eichborn, Investoren als Entwicklungshelfer? Zur Rolle von Krediten, Direktinvestitionen und PortfolioAnlagen in Entwicklungsländern, Berlin, Berliner Landesarbeitsgemeinschaft Umwelt und Entwicklung (BLUE 21) ; Bonn, Evangelischer Entwicklungsdienst, 2006, <http://www.blue21.de> oder $<$ http://www.eed.de>.

31 World Bank, GDF 2004.

32 UNCTAD, WIR 2004, S. 127. Working Group of the Capital Markets Consultative Group, Foreign Direct Investment in Emerging Market Countries, Washington, DC, IMF, September 2003.

33 UNCTAD, Economic Development in Africa: Rethinking the Role of Foreign Direct Investment, New York; Genf, 2005, <http://www.unctad.org>.

34 World Bank, GDF 2003. Robert E. Lipsey, Foreign Direct Investors in Three Financial Crisis, NBER Working Paper, Nr. 8048, Cambridge, MA, National Bureau of Economic Research, 2001. 
dennoch markanten kurzfristigen Kapitalabflüssen und zu noch stärkeren Wechselkursschwankungen beitragen ${ }^{35}$.

Auf einen weiteren, möglicherweise verhängnisvollen Aspekt weist Kavaljit Singh hin. Zunehmend werden direkte Auslandsinvestitionen im Dienstleistungsbereich (z.B. in Telekommunikation, Energie, Bau, Detailhandel, Finanzdienstleistungen etc.) getätigt. Diese Dienstleistungen charakterisieren sich dadurch, dass sie lokal angeboten und konsumiert werden müssen (non tradable goods). Weil aber Investitionen für die lokalen Märkte eher zu kurzfristigen und raschen Rücktransfers führen, könnte sich der Nettoabfluss in diesen Sektoren und betroffenen Ländern verstärken ${ }^{36}$.

Beigetragen zum starken Anstieg von FDI hat auch die Privatisierungswelle staatlicher Unternehmen. Als sich das Angebot an privatisierungswürdigen Unternehmen zu erschöpfen begann, reduzierte sich auch der Umfang der durch Privatisierungen induzierten FDI.

Portfolioinvestitionen schwanken beträchtlich stärker als FDI und verhalten sich prozyklisch, sowohl im Konjunkturaufschwung wie im -abschwung. In den frühen 90er-Jahren überstiegen sie mengenmässig die FDI. Mit der Finanzkrise Asiens fielen sie in sich zusammen. Erst die letzten Jahre brachten wieder einen Aufschwung. Das hängt zumindest zum Teil mit dem Investitionsverhalten institutioneller Anleger zusammen, die vermehrt auch in den sich entwickelnden lokalen Finanzmärkten zu investieren bereit sind ${ }^{37}$.

Problematisch für Entwicklungsländer kann zudem das spekulative Ausnutzen von Zinsdifferenzen zwischen Ländern sein, das sog. „Carry Trading “. Investoren nehmen Kredite in zinstiefen Märkten auf und investieren sie in Hochzinsmärkten oder an boomenden Börsen. Werden die Zinsdifferenzen geringer, dann werden diese spekulativen Anlagen unvermittelt zurückgezogen.

Fazit: Jedem Zustrom von privatem Investitionskapital aus dem Ausland folgt unweigerlich irgendwann ein Kapitalabfluss. Eine transparente Nettotransferstatistik besteht nicht. Sie müsste alle mit Investitionen verbundenen Transfers umfassen. Private Investitionsflüsse als nicht schuldenwirksam zu bezeichnen, rechtfertigt sich nicht.

\section{Schuldenwirksame private Kapitalflüsse}

Bei den privaten schuldenwirksamen Kapitalflüssen ist zwischen Bankkrediten und Obligationenanleihen (Bonds) zu unterscheiden. Beide gelten als mittel- bis langfristige Finanzierungsinstrumente. Hinzu kommen kurzfristige Kredite, meist Handelskredite mit Laufzeiten von maximal zwei Jahren. Sie schrumpften zu Beginn des neuen Jahrtausends beträchtlich und sind erst in den letzten Jahren wieder angestiegen (vgl. Tabelle 3).

35 Ricardo French-Davis und Stephany Griffith-Jones, From Capital Surges to Drought: Seeking Stability for Emerging Economies, Basingstoke (UK), Palgrave Macmillan, 2003 (zitiert nach José Antonio Ocampo et al., op. cit., S. 25).

36 Kavaljit Singh, op. cit., S. $44 \mathrm{ff}$.

37 World Bank, GDF 2006, Vol. 1, S. 53. 
Kurzfristige Kredite reagierten auf die letzte Finanzkrise in der zweiten Hälfte der 90er-Jahre unmittelbar. 1998 flossen allein 66 Milliarden Dollar an kurzfristigen Krediten vom Süden in den Norden zurück. Ab 1998 überstiegen die Rückzahlungen für alle Entwicklungsländer die neu gewährten Kredite. Dieser negative Ressourcenfluss begann sich erst ab dem Jahr 2002 wieder ins Positive zu drehen. Dann stiegen diese kurzfristigen Flüsse rasch auf mittlerweile 70 Milliarden Dollar (2005) an. Dieser Zuwachs konzentriert sich allerdings fast ausschliesslich auf China, Malaysia und andere ostasiatische Länder sowie auf die Türkei, Russland und weitere osteuropäische Länder ${ }^{38}$.

Ein ähnliches Bild stark schwankender $\mathrm{Zu}$ - und Abflüsse lässt sich auch in früheren Zyklen finden. Schwankungen machen insbesondere den Ländern mit niedrigem Einkommen zu schaffen. Weil für sie der Zugang zu den längerfristigen privaten Finanzströmen schwieriger ist, neigen sie eher dazu, zu kurzfristigen Krediten wie Exportkrediten zu greifen.

Mittel- und langfristige Bankkredite stiegen noch bis 1998 an (50 Mrd. \$), ehe die Rückflüsse auch hier die Neuausleihungen überstiegen. Seit 2003 stiegen die Nettoausleihungen (Neuausleihungen abzüglich Rückzahlungen) wieder an, mit einem neuen Rekordhoch im Jahre 2005 (67 Mrd. \$). Diese neue Welle von meist von mehreren Banken gemeinsam gewährten Krediten (Syndikate) verteilte sich auf verschiedenste Sektoren, doch Erdöl- und Erdgasprojekte dominierten. Zehn Länder beanspruchten 70 Prozent dieser Anleihen ${ }^{39}$, während 77 meist ärmste Länder überhaupt keinen Zugang zu solchen Bankkrediten hatten.

Ähnlich verlief die Entwicklung bei den Obligationenanleihen. Neuanleihen überstiegen zwar in den letzten Jahren immer Rückzahlungen, aber der Nettozustrom wurde bis ins Jahr 2002 immer geringer (10 Mrd. \$), ehe er wieder auf mittlerweile 62 Milliarden Dollar (2005) anstieg. Auch hier absorbieren acht Länder mit insgesamt 70 Prozent den Löwenanteil der Obligationenanleihen ${ }^{40}$. Im Jahr 2005 traten vierzig Länder auf den internationalen Anleihensmärkten auf. Die Tendenz ist steigend ${ }^{41}$.

Auch die hier verwendeten Statistiken bezeichnen sich als Nettokapitalflüsse. Erfasst werden Neuschulden sowie Amortisationszahlungen ${ }^{42}$. Sie schliessen allerdings die Zinszahlungen nicht mit ein (siehe unten).

Zwei strukturelle Veränderungen in den privaten internationalen Finanzmärkten können möglicherweise mithelfen, das Volatilitätsproblem zu entschärfen. Einerseits sind einige Schwellenländer heute in der Lage, in ihrer eigenen Währung internationale Anleihen zu begeben. Anderseits verstärkten lokale Filialen internationaler Banken die Kreditgewährung in lokaler Währung. Diese Mittel stammen aus lokal gesammelten Ersparnissen. Dabei wäre allerdings noch die Frage zu klären, ob dies auf Kosten der Tätigkeit einheimischer Banken geschieht, mithin also zu einem Verdrängen (, Crowding out“) lokaler Banken führt.

38 World Bank, GDF 2006, Vol. 2, Tabellen A.1 und A.11.

39 Brasilien, Chile, China, Indien, Mexiko, Polen, Russland, Südafrika, Thailand und die Türkei (World Bank, GDF 2006, Vol. 1, S. 48).

40 Chile, China, Malaysia, Mexiko, Polen, Russland, Thailand und Ungarn (ibid., S. 49).

41 Ibid., S. $45 \mathrm{ff}$.

42 Vgl. dazu ibid., Tabellen A.1 bis A. 15. 
Fazit: Die mit hohen Schwankungen einhergehenden, oft kurzfristigen Kapitalflüsse wirken stets und ausgeprägt prozyklisch. Ein abrupter und hoher Rückfluss von Privatkapital verursacht hohe wirtschaftliche und soziale Kosten. Er trägt zum Rückgang von Produktion und Konsum bei. Der Zugang zu längerfristigen Bankkrediten sowie zu den internationalen Obligationenmärkten ist den ärmsten Ländern faktisch verwehrt.

\section{Schuldenströme und Entschuldung}

Die Schuldenstatistiken sind ernüchternd: Zählt man alle den Entwicklungsländern aus privaten und öffentlichen Quellen, zu kommerziellen und konzessionellen Bedingungen zugeflossenen Kredite zusammen, dann kommt man im Jahr 2005 insgesamt auf 444 Milliarden Dollar Neugeld (ohne IWF). Im gleichen Jahr haben die Entwicklungsländer aber für 393 Milliarden Dollar alte Schulden zurückbezahlt und für 117 Milliarden Dollar Zinsen beglichen. Der gesamte Schuldendienst belief sich demnach auf 511 Milliarden Dollar (gerundeter Betrag). Demzufolge flossen netto 67 Milliarden mehr vom Süden in den Norden $^{43}$ (siehe Tabelle 4). Die Zahlen für die Vorjahre sind nicht grundsätzlich anders. Social Watch hält fest, dass seit 1984 der Schulden-Nettotransfer an Entwicklungsländer mit Ausnahme von drei Jahren stets negativ gewesen sei. Afrika habe seinen Schuldenbestand auf 200 Milliarden Dollar erhöht, obschon der Kontinent seit 1970296 Milliarden der eingegangenen Schulden von 320 Milliarden Dollar beglichen habe ${ }^{44}$.

Mit Ausnahme von Osteuropa/Zentralasien verzeichneten alle Regionen in den letzten Jahren einen negativen Nettoschuldentransfer (Auszahlungen minus Amortisationen minus Zinszahlungen), selbst Afrika südlich der Sahara und die Gruppe der ärmsten Länder insgesamt. Während bei Schwellenländern und Ländern mit mittlerem Einkommen in der Regel der Anteil der privaten Schulden höher ist als jener der öffentlichen Schulden, ist es bei den ärmsten Ländern gerade umgekehrt.

Tabelle 4: Schuldenbestand und Schuldenflüsse, 2005 (in Milliarden Dollar)

\begin{tabular}{lcccrrr}
\hline & $\begin{array}{c}\text { Schulden- } \\
\text { bestand }\end{array}$ & $\begin{array}{c}\text { Neu- } \\
\text { Kredite }\end{array}$ & $\begin{array}{c}\text { Amorti- } \\
\text { sations- } \\
\text { zahlungen }\end{array}$ & $\begin{array}{c}\text { Zinsen } \\
\text { Schulden- } \\
\text { dienst }\end{array}$ & $\begin{array}{c}\text { Schulden- } \\
\text { netto- } \\
\text { tranfer }\end{array}$ \\
\hline Entwicklungsländer total & 2800 & 444 & 393 & 117 & 511 & -67 \\
\hline Ostasien+Pazifik & 634 & 75 & 61 & 21 & 82 & -7 \\
\hline Europa+Zentralasien & 870 & 197 & 147 & 34 & 181 & +16 \\
\hline Lateinamerika+Karibik & 724 & 117 & 135 & 43 & 178 & -61 \\
\hline Mittlerer Osten+Nordafrika & 162 & 16 & 15 & 6 & 21 & -5 \\
\hline Südasien & 195 & 25 & 21 & 5 & 26 & -1 \\
\hline Afrika südlich der Sahara & 180 & 15 & 16 & 8 & 23 & -8 \\
\hline Ärmste Länder & 412 & 36 & 31 & 12 & 43 & -7 \\
\hline Länder mit mittlerem Einkommen & 388 & 408 & 362 & 105 & 468 & -60 \\
\hline
\end{tabular}

Quelle: World Bank, GDF 2006, Vol. 2, Summary Tables

43 World Bank, GDF 2006, Vol. 2, Summary Tables. Der Bericht enthält detaillierte Zahlen für die einzelnen Länder.

44 Sony Kapoor, „Exposing the Myth and Plugging the Leaks“, Social Watch Report 2006, Impossible Architecture, Montevideo, 2006, S. 17 ff., <http://www.socialwatch.org>. 
Ein hoher Anteil dieser Schulden wurde zur Finanzierung von schlecht vorbereiteten oder durchgeführten Projekten verwendet oder verschwand in die Taschen korrupter Regimes. Der Beispiele gibt es viele ${ }^{45}$. Dies trug zur Kapitalflucht bei (siehe unten).

Die untragbare Schuldenlast führte zur Forderung nach Schuldenstreichung. Die wohl bekanntesten und am meisten diskutierten Entschuldungsinitiativen der letzten Jahre sind die Initiative von IWF und Weltbank zugunsten der am stärksten verschuldeten ärmsten Länder (HIPC-Initiative), ergänzt durch die von den grossen Ländern (G-8) vor zwei Jahren gestartete multilaterale Entschuldungsinitiative (MDRI). Sie entlasteten nur jene hochverschuldeten ärmsten Entwicklungsländer, die sich mit ihrer Politik den strengen Anforderungen von IWFund IDA-Programmen unterzogen. Bis Ende 2006 konnten sich dreissig Länder für die HIPC qualifizieren ${ }^{46}$. Davon haben 22 Länder den ganzen Prozess vollzogen, acht Länder stecken im Verfahren. Zehn weitere Länder sind potenziell qualifizierbar. Wenn alle dreissig Länder den vollständigen Prozess durchlaufen haben, wird ihr Schuldenbestand (als Nettogegenwartswert verstanden) um zwei Drittel reduziert sein. Der Anteil des Schuldendienstes an den Erlösen von Exporten und Dienstleistungen sollte dann von durchschnittlich 16 Prozent auf 8 bis 9 Prozent reduziert werden. Die dadurch frei werdenden Mittel sollen in die Armutsbekämpfung fliessen. In diesen Ländern ist denn auch der Anteil der Sozialausgaben am BIP zwischen 1999 und 2005 von 6 auf 9 Prozent gestiegen.

Mit der multilateralen Entschuldungsinitiative (MDRI) sollen die Restschulden der HIPC-Länder gegenüber IWF, Weltbank (IDA) und dem Afrikanischen Entwicklungsfonds ebenfalls erlassen werden, wobei die Geberstaaten dazu den Entwicklungsbanken Kompensationszahlungen leisten müssten. Der IWF deckt die Kosten aus eigenen Mitteln. Im Frühjahr 2007 schloss sich die Interamerikanische Entwicklungsbank faktisch für ihre fünf ärmsten Länder der MDRI an. Mit der MDRI sollen rund 50 Milliarden Dollar zusätzlich gestrichen werden. Das wird den Schuldendienst zu Beginn jährlich um eine Milliarde Dollar, in einer späteren Phase sogar um annähernd zwei Milliarden entlasten.

Diese Entlastungen können jedoch von Land zu Land deutlich variieren. Nicht bei allen Ländern hat die HIPC-Initiative dazu geführt, dass sie ihre Schuldenlast auf ein tragbares Niveau absenken konnten. Eine Evaluation der Weltbank zeigte, dass sich elf von dreizehn Ländern seit dem Ablauf des HIPC-Prozesses erneut mit sich verschlechternden Schuldenindikatoren ${ }^{47}$ konfrontiert sehen. Immerhin wird anerkannt, dass die HIPC-Initiative positive Auswirkungen auf den Entwicklungsprozess haben kann.

45 Vgl. z.B. Eurodad, Skeletons in the Cupboard: Illegitime Debt Claims of the G7, 2007. Eurodad publiziert neu auch ein Illegitimate Debt Campaign Bulletin, <http://www.eurodad.org>. Christian Aid, Enough is Enough: The Debt Repudiation Option, London, 2007, <http://www.christianaid.org>.

46 Zur Heavily Indebted Poor Country Initiative (HIPC) und zur Multilateral Debt Relief Initiative (MDRI) siehe <http://www.worldbank.org/hipc> oder <http://www.worldbank.org/economicpolicyanddebt $>$ und <http://www.imf.org/external/np/exr/facts/hipc.htm $>$. Dort ist auch eine detaillierte Liste der Länder verfügbar. Für Informationen über die laufende Umsetzung der Initiativen vgl. auch das Kapitel „Internationale Finanzbeziehungen“ im Band Fakten und Statistiken des Schweizerischen Jahrbuchs für Entwicklungspolitik (IUED, Genf), diverse Jahrgänge.

47 Übliche Schuldenindikatoren sind etwa das Verhältnis der Schulden zum BIP, der Schulden zum Export, des Schuldendienstes zum Export oder des Schuldendienstes zu den Staatsausgaben. 
Die UNO schreibt, dass die meisten HIPC-Länder trotz Entschuldungsmassnahmen weit davon entfernt seien, genügend Mittel zum Erreichen der Millenniumsziele aufzuweisen ${ }^{48}$. Sie kritisiert auch, dass die ursprüngliche Forderung, wonach die Entschuldung durch zusätzliche Mittel zu finanzieren sei, bei weitem nicht erreicht worden sei.

Weil die Entschuldungsmassnahmen im Rahmen von HIPC und MDRI nur die ärmsten Länder betreffen, sind die Auswirkungen auf die Gesamtschulden aller Entwicklungsländer gering. Die Gesamtschuld ist allerdings in den letzten Jahren nur noch langsam angestiegen und erreichte Ende 2005 die Höhe von 2200 Milliarden Dollar. Der Schuldenbestand der ärmsten Länder betrug 412 Milliarden, jener der Staaten Afrikas südlich der Sahara immer noch 181 Milliarden.

Die Schulden der Entwicklungsländer gegenüber den offiziellen bilateralen Gläubigern werden im sog. Pariser Klub behandelt ${ }^{49}$. Ein grosser Teil dieser Schulden ist auf öffentlich garantierte Exportkredite der privaten Exportwirtschaft zurückzuführen. Faktisch entspricht dies einer Übernahme privater Kredite durch die öffentliche Hand. Weil die Schuldner sehr oft keinen Schuldendienst mehr leisteten, wurden Zahlungsrückstände kapitalisiert, so dass der Schuldenbestand ständig weiter anstieg.

Der Pariser Klub beschäftigt sich sowohl mit den Schulden der ärmsten Länder wie auch mit jenen der Länder mit mittlerem Einkommen. Für die Qualifikation zur HIPC-Initiative benötigen die ärmsten Länder zuerst ein Abkommen mit dem Pariser Klub. Bei den Umschuldungen von Ländern mit mittlerem Einkommen verbesserte der Pariser Klub im Laufe der Jahrzehnte die Umschuldungsbedingungen stetig, letztmals mit dem sog. Evian-Prinzip (genannt nach dem G-8Gipfel von 2003 in Evian). Danach sollen die länderspezifischen Umstände angemessener berücksichtigt werden und eine Mischung aus Aufschub von Schuldendienstzahlungen und Teilstreichung des Schuldenbestands angewendet werden, damit auch Länder mit mittleren Einkommen mittelfristig ein tragbares Schuldenniveau erreichen können ${ }^{50}$. Bislang sind einzig die Schulden des Irak und Nigerias gemäss diesem Ansatz behandelt worden. Der Irak erreichte eine Schuldenreduktion von 80 Prozent, Nigeria eine zweimalige Reduktion um ein Drittel, nachdem das afrikanische Ölland vorher all seine Zahlungsrückstände beglichen hatte. Das bewirkte letztlich eine Entschuldung von 18 Milliarden Dollar.

Schulden gegenüber privaten Gläubigern sind in der Vergangenheit stets von Fall zu Fall behandelt worden, sei es früher in einem informellen „Londoner Club" weltweit tätiger führender Grossbanken, sei es durch marktnahe Lösungen, sei es durch die von früheren US-Regierungen initiierten Pläne: Baker-Plan (1985) und Brady-Plan (1989).

Offensichtlich ist es schwierig, den Umfang aller Entschuldungsmassnahmen zu quantifizieren. Einerseits sind die statistischen Unterlagen lückenhaft, andererseits sind die Definitionskriterien für Entschuldungsmassnahmen zwischen den verschiedenen Gläubiger- und Schuldnerkategorien zu unterschiedlich. James Henry bezeichnet die Summe aller Entschuldungsmassnahmen jedoch als

48 United Nations, WESP 2007, S. 72.

$49<$ http://www.clubdeparis.org $>$.

50 Vgl. José Antonio Ocampo et al., op. cit., S. 119 ff. 
bescheiden $^{51}$. Gesamthaft seien den Entwicklungsländern zwischen 1982 und 2005 lediglich 310 Milliarden Dollar Schulden gegenüber öffentlichen und privaten Gläubigern gestrichen oder umgeschuldet worden. Das entspreche gerade noch 7,8 Prozent der ausstehenden gesamten Schuldenlast per Ende 2005 von 4000 Milliarden Dollar (Nettogegenwartswerte). Für die ärmsten Länder (LICs) betrage die Entlastung immerhin 28 Prozent (vgl. Tabelle 5).

Tabelle 5: Entschuldungsmassnahmen, 1982-2005 (in Milliarden Dollar, Nettogegenwartswerte)

\begin{tabular}{lccr}
\hline & $\begin{array}{c}\text { Länder mit } \\
\text { niedrigem } \\
\text { Einkommen }\end{array}$ & $\begin{array}{c}\text { Länder mit } \\
\text { mittlerem } \\
\text { Einkommen }\end{array}$ & Total \\
\hline Schuldenbestand vor Entschuldung & 574 & 3426 & 4000 \\
\hline Entschuldung & 161 & 149 & 310 \\
\hline Schuldenbestand nach Entschuldung & 413 & 3277 & 3690 \\
\hline Entschuldung (in \% des Bestands) & 28.1 & 4.3 & 7.8 \\
\hline
\end{tabular}

Quelle: James S. Henry, "The Mirage of Debt Relief”, in Steven Hiatt (ed.), A Game as Old as Empire: The Secret World of Economic Hit Men and the Web of Global Corruption, San Francisco, Berrett-Koehler, 2007, aufgrund von Weltbankzahlen, eigene Berechnungen von James Henry.

Fazit: Die Verschuldung hat letztlich nicht dazu beigetragen, dass die Entwicklungsländer mehr Mittel für die Finanzierung ihrer Entwicklung zur Verfügung haben; sie hat vielmehr zu einem stetigen Mittelabfluss geführt. Entschuldungsmassnahmen schlugen nur bei den ärmsten Ländern massgeblich zu Buche. Was die Situation noch schwieriger macht, ist die Tatsache, dass ein grosser Teil dieser Schulden von Diktatoren und Kleptokraten zu ihrer persönlichen Bereicherung eingegangen worden ist. Ein anderer bedeutender Teil floss in schlechte Projekte.

\section{Entwicklungs- und Schwellenländer sind Nettokapitalexporteure}

Nach den Textbüchern der ökonomischen Theorie müsste Kapital eigentlich aus den kapitalreichen, entwickelten Ländern in die kapitalarmen Entwicklungsländer fliessen. Die bisherigen Ausführungen zeigen jedoch, dass das Gegenteil der Fall ist. Stellt man auf die Bilanz der laufenden Zahlungen (Current account balance) ab, wie dies u.a. der IWF und die UNO tun ${ }^{52}$, sind die Entwicklungsländer heute insgesamt Nettokapitalexporteure, einige Industrieländer sind Nettokapitalimporteure.

Grösster Kapitalimporteur sind die USA. Grosse Kapitalimporteure sind ebenfalls Australien, England und Spanien. Kumuliert beträgt das Defizit der Bilanz der laufenden Zahlungen dieser entwickelten Länder Ende 2006 über 600

51 James S. Henry, „,The Mirage of Debt Relief“, in Steven Hiatt (ed.), A Game as Old as Empire: The Secret World of Economic Hit Men and the Web of Global Corruption, San Francisco, Berrett-Koehler, 2007, S. 225. Der Autor stützt sich zwar auf Weltbankdaten, ergänzt sie allerdings mit Daten für Länder, die nicht in den Weltbankstatitstiken eingeschlossen sind. Zudem verwendet er Nettogegenwartswerte und kommt deshalb zu beträchtlichen Abweichungen im gesamten Schuldenbestand, verglichen mit der Weltbank.

52 IMF, World Economic Outlook (nachstehend WEO), Tabelle 32, „Summary of Balance of Payments, Capital Flows, and External Financing“, div. Ausgaben, <http://www.imf.org/weo >. United Nations, WESP 2007, S. 32 ff. 
Milliarden Dollar. Sehr viele Entwicklungsländer weisen hingegen heute einen Überschuss in ihrer Bilanz der laufenden Zahlungen auf.

Grafik 2: Wichtigste Nettokapitalexport- und -importländer, 2006
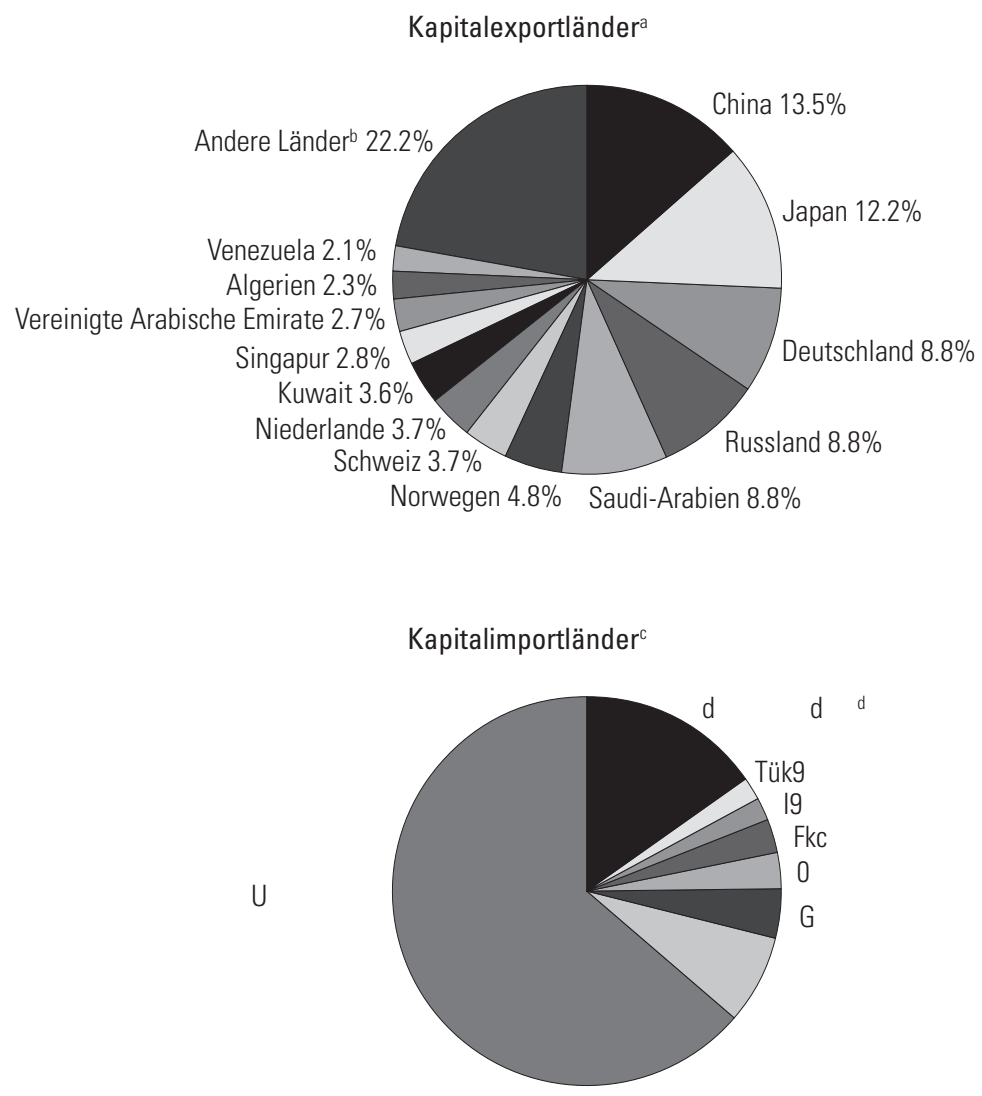

Quelle: IMF, Global Financial Stability Report: Market Developments and Issues, April 2007, <http://www.imf.org/external/ pubs/ft/GFSR/index.htm>, S. 141.

a Gemäss Leistungsbilanzüberschuss (Current account surplus) der Länder (unter Annahme, dass „ungeklärte Beträge“ (errors and omissions) Teil der Kapital- und Finanzkonten sind).

b Unter „Andere Länder” fallen alle Länder, deren Anteile am Gesamtüberschuss unter 2,1% liegen.

c Gemäss Leistungsbilanzdefizit (Current account deficit) der Länder (unter Annahme, dass „ungeklärte Beträge” (errors and omission) Teil der Kapital- und Finanzkonten sind).

d Unter „Andere Länder" fallen alle Länder, deren Anteile am Gesamtdefizit unter 1,9\% liegen.

Die UNO erstellte auf Grund von Zahlen des IWF eine Nettotransferrechnung von finanziellen Ressourcen ${ }^{53}$. Diese Rechnungen addieren alle Zuflüsse aus Investitionen, Hilfe und Schuldenflüssen und ziehen davon alle Abflüsse für Zinsen und Gewinne sowie die Erhöhung internationaler Währungsreserven und die Kapitalanlagen von Privaten im Ausland ab. Der Nettofinanztransfer eines

53 United Nations, WESP 2007, S. 57 ff. Die IWF-Zahlen sind der World Economic Outlook Database und den Balance of Payment Statistics entnommen. Vgl. dazu auch die Tabelle 32, ,Summary of Balance of Payments, Capital Flows, and External Financing“, in IMF, WEO, September 2006, und deren Fussnote 6. 
Landes entspricht grundsätzlich und mit umgekehrten Vorzeichen dem Saldo aus der Bilanz aller Exporte und Importe von Gütern und Dienstleistungen.

Ein Überschuss in dieser Leistungsbilanz entsteht, wenn die einheimische Produktion den einheimischen Konsum und die einheimischen Investitionen übersteigt und dieser Überschuss im Ausland (statt im Lande selber) investiert wird. Ein Nettofinanztransfer der Entwicklungsländer hilft mit, das Zahlungsbilanzdefizit insbesondere der USA zu finanzieren, deren Konsum und Investitionen über ihren Ersparnissen liegen. Mit anderen Worten: Der Süden finanziert die USA, die über ihre Verhältnisse leben und 70 Prozent der weltweiten, externen Ersparnisse absorbieren.

In dieser Nettofinanztransfer-Rechnung weisen die Entwicklungsländer insgesamt seit zehn Jahren und die Transitionsländer seit acht Jahren stets einen Nettoabfluss auf, der sich vor allem in den letzten Jahren noch stark akzentuierte. Afrika (ohne Südafrika und Nigeria) verzeichnete bis 2005 einen Nettozufluss, allerdings mit abnehmender Tendenz. Dasselbe trifft für die Gruppe der ärmsten Länder (LICs) zu. Das ist hauptsächlich auf den Zustrom der öffentlichen Hilfe zurückzuführen. Im Jahr 2006 haben aber auch Afrika und die Gruppe der Länder mit niedrigem Einkommen (LICs) einen Nettoabfluss ausgewiesen. Einzig die HIPC-Länder profitierten auch noch im Jahr 2006 von einem positiven Zufluss (vgl. Tabelle 6).

Tabelle 6: Nettofinanztransfers an Entwicklungs- und Transitionsländer, 1995-2006 (in Milliarden Dollar)

\begin{tabular}{|c|c|c|c|c|c|c|c|c|c|c|c|c|}
\hline & 1995 & 1996 & 1997 & 1998 & 1999 & 2000 & 2001 & 2002 & 2003 & 2004 & 2005 & $2006^{p}$ \\
\hline $\begin{array}{l}\text { Entwicklungs- } \\
\text { länder }\end{array}$ & 46.2 & 24.4 & -0.6 & -33.8 & -115.9 & -178.2 & -151.9 & -202.7 & -295.6 & -346.8 & 525.0 & -657.7 \\
\hline $\begin{array}{l}\text { Afrika südl. } \\
\text { Sahara }^{a}\end{array}$ & 7.5 & 5.4 & 7.6 & 12.1 & 8.9 & 2.8 & 7.0 & 5.3 & 6.8 & 4.5 & 0.6 & -10.6 \\
\hline $\begin{array}{l}\text { Ost- und } \\
\text { Südasien }\end{array}$ & 21.8 & 18.8 & -31.9 & -128.4 & -137.6 & -119.1 & -115.6 & -146.0 & -170.7 & -162.1 & -230.5 & -244.7 \\
\hline Westasien & 20.1 & 10.6 & 12.6 & 34.8 & 7.7 & -29.7 & -23.8 & -18.4 & -43.3 & -69.8 & -125.9 & -194.7 \\
\hline $\begin{array}{l}\text { Latein- } \\
\text { amerika }\end{array}$ & -1.7 & 0.6 & 23.4 & 44.3 & 9.8 & -1.6 & 4.3 & -31.6 & -60.6 & -80.0 & -150.2 & -123.1 \\
\hline $\begin{array}{l}\text { Transitions- } \\
\text { länder }\end{array}$ & -2.7 & -6.3 & 2.8 & 3.6 & -23.7 & -49.4 & -29.1 & -26.1 & -33.7 & -54.6 & -86.8 & -125.1 \\
\hline HIPCs & 6.7 & 7.0 & 7.4 & 8.5 & 9.7 & 7.8 & 8.2 & 10.3 & 9.6 & 10.2 & 11.5 & 9.8 \\
\hline LICs & 11.8 & 10.4 & 9.3 & 12.5 & 110.8 & 5.7 & 8.8 & 7.1 & 8.5 & 5.4 & 2.6 & -4.3 \\
\hline
\end{tabular}

Quelle: United Nations, World Economic Situation and Prospects 2007, New York, 2007, Tabelle III.1, S. 58, basierend auf Zahlen des IWF. Die Ländergruppen sind allerdings anders aggregiert als in den IWF-Statistiken.

Anmerkung: Ein Minus (-) entspricht einem Netto-Finanztransferabfluss, ein Plus (+) einem Netto-Finanztransferzufluss.

p Provisorische Schätzung.

a Ohne Südafrika und Nigeria.

Ein Nettokapitalexport kann darauf zurückgeführt werden, dass das Finanz- und Bankensystem vieler Entwicklungsländer noch ungenügend entwickelt ist, überschüssiges Kapital also nicht wieder produktiven Zwecken im Inland zugeführt werden kann ${ }^{54}$. Als Ursache des negativen Transfers erachtet Jubilee Research

54 Vgl. etwa Raghuram Rajan, Financial System Reform and Global Current Account Imbalances, Remarks at the American Economic Association (AEA) meetings, Boston (MA), 8. Januar 2006, $<$ http://www.imf.org/external/np/speeches/2006/010806.htm> (R. Rajan war damals Director of Research des IWF). 
bei den Ländern mit mittlerem Einkommen (vor allem in Lateinamerika) hingegen die Zinszahlungen auf den Schulden und die Gewinnrücktransfers auf den FDIs. Bei den Schwellenländern, vor allem bei China, hänge der Abfluss in erster Linie vom Aufbau von Guthaben im Ausland, insbesondere vom Ausbau der internationalen Währungsreserven $a b^{55}$.

Mit diesem Kapitalexport konnten die meisten Entwicklungs- und Transitionsländer ihre internationalen Währungsreserven in den vergangen zehn Jahren sowohl in absoluten Zahlen wie auch im Verhältnis zum Niveau ihrer Importe erhöhen ${ }^{56}$. Ursprünglich entsprang der Aufbau grösserer internationaler Währungsreserven dem Gedanken eines Selbstschutzes gegen Finanzkrisen. Das entspricht einem rationalen Denken der betroffenen Länder, zumal die vom internationalen System zur Verfügung gestellten Abwehrmechanismen nur beschränkt $\operatorname{sind}^{57}$. Mittlerweile scheinen allerdings die angehäuften Reserven die für den Selbstschutz notwendige Höhe deutlich zu übersteigen. Das stellt Fragen nach Nutzen und Kosten, zumal wenn diese Reserven mehrheitlich in US-Dollar angelegt sind, einer Währung, die einem grossen Abwertungsrisiko ausgesetzt ist.

Der Kapitalzufluss der letzten Jahre in die Entwicklungsländer, insbesondere der starke Anstieg privater Kapitalzuflüsse, hat gemäss Weltbank aber nicht nur zur Folge gehabt, dass diese Länder damit ihre internationalen Währungsreserven substanziell erhöhen konnten. Statt lokale Investitionen anzukurbeln, hätte dies andererseits privaten Unternehmungen und Individuen vermehrt die Möglichkeit gegeben, Anlagen im Ausland zu tätigen, kommentiert die Weltbank ${ }^{58}$. Die Weltbank veranschlagte diesen Kapitalexport von Privaten im Jahre 2005 auf 258 Milliarden Dollar. Diese Summe sei zwar schwierig zu interpretieren, weil sie aus dem Posten „Fehler und Auslassungen“ (Errors and Omissions) der Zahlungsbilanzstatistiken hergeleitet sei ${ }^{59}$. Sprechend sind dafür die Zahlen, welche die Weltbank als „Balancing Items “ aufführt, eine Kombination von „Errors and Omissions " und den Nettoakquisitionen von ausländischen Guthaben durch Entwicklungsländer. Dieser Posten belief sich im Jahr 2005 auf 272 Milliarden Dollar (Vorjahr: 121 Mrd. \$). Wie viel davon der ominösen Kapitalflucht zuzuschreiben ist, bleibt ungeklärt.

Fazit: Rechnet man alle Finanzströme auf, so transferieren die Entwicklungsländer heute insgesamt netto Kapital in die reichen Länder, hauptsächlich in jene Länder mit Defiziten in ihren Zahlungsbilanzen. Das ist Ausdruck der Anhäufung internationaler Währungsreserven durch die Entwicklungsländer. Aber es umfasst auch die zunehmenden Anlagen Privater aus Entwicklungsländern auf den weltweiten Kapitalmärkten, ermöglicht durch die Kapitalverkehrsliberalisierung der letzten Jahre. Das hat auch der Kapitalflucht Auftrieb gegeben.

55 Jubilee Research, Water Flowing uphill: Why South-North Financial Flows Defy Conventional Logic, $<$ http://www.jubileeresearch.org/news/SNFinFloGKN1a.pdf>.

56 Vgl. dazu IMF, WEO, Statistical Appendix, Tabelle 35, „Reserves“, September 2006.

57 José Antonio Ocampo et al., op. cit., S. 3 und S. 21 ff.

58 World Bank, GDF 2006, Vol. 1, S. 4 und Abb. 3.

59 Der IWF fügt in der Erklärung zu diesen Posten „Errors and Omissions“ in Klammern folgende Bemerkung hinzu: ,not infrequently reflecting capital flight“ (,nicht selten Kapitalflucht widerspiegelnd“, unsere Übersetzung, AdR). Siehe IMF, WEO, „Statistical Appendix“, Tabelle 32, Fussnote 6, September 2006. 
Versuche, den schillernden Begriff „Kapitalflucht“ klar zu definieren und den Umfang zu messen, gibt es viele ${ }^{60}$. Raymond Baker veranschlagt das Ausmass der weltweiten grenzüberschreitenden schmutzigen Geldflüsse auf mindestens 1000 Milliarden Dollar, wobei die Hälfte davon aus Entwicklungs- und Transitionsländern stamme ${ }^{61}$. Der Africa Report 2006, von der Zeitschrift Jeune Afrique herausgegeben, schrieb, die Kapitalflucht aus Afrika belaufe sich heute auf über 30 Milliarden Dollar pro Jahr ${ }^{62}$. Zum Vergleich: Die gesamte öffentliche Entwicklungshilfe an Afrika beträgt gemäss OECD 25 Milliarden Dollar. Die beiden afrikanischen Forscher James Boyce und Léonce Ndikumana schätzen, dass dreissig afrikanische Länder zwischen 1970 und 1996 insgesamt 187 Milliarden Dollar an Fluchtkapital verloren hätten ${ }^{63}$. Die Ausgabe der Zeitschrift Marchés tropicaux et méditerranéens vom 4. Mai 2007 geht noch weiter. Danach betrug die Kapitalflucht aus Afrika südlich der Sahara in den 90erJahren insgesamt 274 Milliarden Dollar ${ }^{64}$. Zum Vergleich: Die Schulden Afrikas betrugen Ende 2005215 Milliarden Dollar. Eine britische parlamentarische Kommission schätzte 2006, Afrikas Elite halte 700 bis 800 Milliarden Dollar in den Finanzzentren des Auslandes ${ }^{65}$. Die Höhe der Zahlen hängt davon ab, wie weit oder eng der Begriff „Kapitalflucht“ gefasst wird.

Unabhängig davon bleibt die Feststellung, dass die grenzüberschreitenden Transfers von schmutzigem Geld in den letzten Jahren zugenommen haben. Diese Kapitaltransfers werden in den üblichen Statistiken nicht oder nur teilweise, mit falschen Werten, erfasst. Raymond Baker erachtet knapp ein Drittel des aus Entwicklungs- und Transitionsländern hinausfliessenden schmutzigen Geldes als kriminell, d.h. aus Handel mit Drogen, Waffen und Menschen stammend, aus Schmuggel, Fälschungen etc. Die anderen zwei Drittel schreibt er kommerziellen Missbräuchen wie falschen Verrechnungspreisen (Transfer Pricing) oder gefälschten Rechnungen $\mathrm{zu}^{66}$.

Zwei Drittel des Welthandels spielen sich nicht auf freien Märkten ab, sondern widerspiegeln konzerninterne Transaktionen (Güter, Kredite, Dienstleistungen, Patente, Royalties, Beratung usw.) von einer Konzerngesellschaft zur anderen. Das eröffnet Spielraum für Preismanipulationen. Werden Exporte preislich unterfakturiert, so fallen die Gewinne im exportierenden Land tiefer aus. Damit

60 Siehe z.B. Edsel L. Beja, „Capital Flight: Meanings and Measures“, in Gerald A. Epstein (ed.), Capital Flight and Capital Controls in Developing Countries, Northampton (MA), Edward Elgar, 2005. Benu Schneider, Measuring Capital Flight: Estimates and Interpretation, ODI Working Paper, Nr. 194, London, Overseas Development Institute (ODI), März 2003, <http://www.odi.org.uk>. V. Cerra, M. Rishi und S.C. Safena, Robbing the Riches: Capital Flight, Institutions and Instability, IMF Working Paper, Nr. 199, Washington, DC, 2005, <http://www.imf.org>.

61 Raymond W. Baker, Capitalism's Achilles Heel: Dirty Money and How to Renew the Free-Market System, Hoboken (NJ), John Wiley, 2005.

62 Patrick Smith, „On the Wing: How Capital Flight Is Cheating Africa“, The Africa Report, Nr. 2, März 2006, <http://www.capitalismsachillesheel.com/AfricaReportArticle.pdf>

63 James K. Boyce und Léonce Ndikumana, „Africa’s Debt: Who Owes Whom?“, in Gerald A. Epstein (ed.), op. cit., Kapitel 13.

64 Dossier fiscalité, Marchés tropicaux et méditerranéens, op. cit.

65 Africa All Party Parliamentary Group, The Other Side of the Coin: The UK and Corruption in Africa, London, März 2006, <http://www.africaappg.org.uk>.

66 Raymond W. Baker, op. cit., S. 172, Tabelle 4.4. 
sinken auch die Steuereinnahmen im betreffenden Land. Werden umgekehrt Importe überfakturiert, dann entstehen im importierenden Land höhere Kosten. Auch das reduziert die Gewinne und die Steuern. Auf diese Weise lassen sich Gewinne auf versteckte Art in Steuerparadiese transferieren, wo keine oder nur geringe Steuern zu entrichten sind ${ }^{67}$.

Wie gross die durch diese Preismanipulationen entstehenden Verluste bei den Steuereinnahmen sind, ist nicht bekannt. Raymond Baker schätzt, 60 Prozent des Handels mit Afrika werde mit falschen Verrechnungspreisen abgewickelt, wobei die Rechnungssumme im Schnitt um elf Prozent von realen Preisen abweichen würde. Ähnliche Grössenordnungen seien in Lateinamerika üblich ${ }^{68}$.

Ernst \& Young befragte im Jahr 2005 Konzerne, bei denen die Transferpreise im Rahmen von Steuerprüfungen untersucht wurden. 44 Prozent der Mutterkonzerne und 34 Prozent der Tochtergesellschaften gaben an, sie hätten nach der Steuerprüfung ihre Buchführung korrigieren müssen, weil sie falsche Preise verwendet hätten ${ }^{69}$.

Zunehmend mehr Regierungen und Steuerbehörden nehmen die Praxis der manipulierten Verrechnungspreise immer stärker unter die Lupe. Noch vor zehn Jahren existierten nur in wenigen Ländern entsprechende Regelungen. Inzwischen kennen 32 Länder solche Vorschriften, weitere prüfen die Einführung ${ }^{70}$.

Transnationale Konzerne nützen ihre Macht auch aus, um von ihren Gastländern Konzessionen in der Besteuerung zu erhalten. Entwicklungsländer sind für weitgehende steuerliche Konzessionen besonders anfällig. Ihre Regierungen senken die Steuersätze. Das britische Hilfswerk Oxfam veranschlagte für die Entwicklungsländer schon im Jahr 2000 einen Ausfall von Steuereinnahmen von 35 Milliarden Dollar pro Jahr ${ }^{71}$. Entwicklungsländer garantieren ausländischen Investoren Steuerbefreiung für fünf oder zehn Jahre, gewähren grosszügige Abschreibungsmöglichkeiten oder andere steuerliche Erleichterungen.

Fazit: Die immer grösser werdende Komplexität von Trusts, Stiftungen und Unternehmen in Offshorezentren bieten immer mehr Möglichkeiten zu legalen, halblegalen und auch illegalen Finanztransfers. Entwicklungsländer mit schwachen Behörden haben diesen Machenschaften wenig entgegenzusetzen. Die Grauzone zwischen legalem und illegalem Handeln wird verschwommen. Das führt zu einem beträchtlichen Verlust an Steuereinnahmen und zu Kapitalflucht.

67 Beispiele von extrem abnormaler Preisgestaltung listen Simon J. Pak (Penn State University) und Mitautorinnen in verschiedenen Publikationen auf. Vgl. z.B. Maria E. de Boyrie, Simon J. Pak und John S. Zdanowicz, „Money Laundering and Income Tax Evasion: The Determination of Optimal Audits and Inspections to Detect Abnormal Prices in International Trade“, Journal of Financial Crime, Vol. 12, Nr. 2, 2004, S. 123-130.

68 Raymond W. Baker, op. cit., S. 170.

69 Siehe auch: Tax Justice Network (TJN), Closing the Floodgates: Collecting Tax to Pay for Development, Paper commissioned by the Norwegian Ministry of Foreign Affairs, chapter 4, „How Companies Reduce Their Tax bills“, London, 2007, S. 42-56, <http://www.taxjustice.net>.

70 Jens Martens, Die öffentliche Armut der Entwicklungsländer, 2006, <http://www.nordsuednetz.de>.

71 Oxfam, Tax Havens: Releasing the Hidden Billions for Poverty Eradication, Oxfam Briefing Papers, Oxford, 2000, <http://www.oxfam.org.uk>. 
Die bisherigen Ausführungen sind nicht als Plädoyer gegen die Entwicklungshilfe zu betrachten, ganz im Gegenteil. Die Hilfeleistungen sind ungenügend. Die öffentliche wie die private Entwicklungshilfe muss deshalb auch in Zukunft beträchtlich erhöht werden. Ohne höhere Entwicklungshilfe können die Millenniumsentwicklungsziele nicht erreicht werden. Aber auch die Qualität der Hilfe bedarf einer weiteren Verbesserung.

Eine Steigerung der Entwicklungshilfe macht aber wenig Sinn, wenn nicht gleichzeitig der enorme Finanzabfluss vom Süden in den Norden gebremst und umgekehrt wird. Die internationale Politik muss also nicht nur auf die Zuflüsse, sondern auch auf die Abflüsse achten.

Fliesst mehr Kapital vom Süden in den Norden als umgekehrt, so beeinträchtigt und unterminiert dies die Mobilisierung lokaler Ressourcen. Es verhindert die Finanzierung von notwendigen Investitionen durch lokale Mittel. Es schwächt das Wachstum und macht die Entwicklungsländer noch abhängiger vom Zufluss externer und oft stark schwankender und schwer voraussehbarer Mittel. Der enorme Kapitalabfluss erklärt zumindest teilweise, weshalb die Armut in der Welt bislang nicht erfolgreicher bekämpft werden konnte.

Von überragender Bedeutung ist das Schliessen der illegalen Abflussschleusen. Das ist nicht nur eine Frage der guten Regierungsführung und der Bekämpfung der Korruption in den Entwicklungsländern selber. Vielmehr darf das internationale Finanzsystem nicht mehr als williger Helfer für illegale Geschäfte dienen. Die reichen Länder und die grossen Finanzplätze haben bislang bei weitem nicht genügend Anstrengungen unternommen, um missbräuchliche Praktiken zu stoppen und illegale Kapitalflucht zu unterbinden. Letztlich sind solche Schritte auch eine Frage der Kohärenz in der Politik der Geberstaaten, in denen sich die grossen Finanzplätze befinden.

Der IWF und die multilateralen Entwicklungsbanken müssen so ausgestaltet werden, dass ihre Politik den Bedürfnissen der Entwicklungsländer dient. Es ist beispielsweise unsinnig, dass die Entwicklungsländer riesige internationale Währungsreserven anhäufen müssen, bloss weil internationale Mechanismen zum Schutz gegen Finanzkrisen ungenügend sind, oder weil deren Inanspruchnahme mit unangemessenen Bedingungen verknüpft ist. Ebenso dringend wären Massnahmen bei der Verschuldung, welche nicht nur die Schuldner, sondern auch die Gläubiger zur Verantwortung ziehen.

Bei den privaten Kapitalflüssen sind Massnahmen gefragt, die zu einer grösseren Stabilität der Zuflüsse führen und abrupte Rückflüsse verhindern. Statistiken, welche vollständige $\mathrm{Zu}$ - und Abflüsse einander gegenüberstellen, werden grössere Transparenz erlauben. 


\section{Bibliografie}

Action Aid International, Real Aid: An Agenda for Making Aid Work, Johannesburg, 2005, $<\mathrm{http}$ ///www.actionaid.org.uk>.

Baker, Raymond W., Capitalism's Achilles Heel: Dirty Money and How to Renew the Free-Market System, Hoboken (NJ), John Wiley, 2005.

Beja, Edsel L., „Capital Flight: Meanings and Measures“, in Gerald A. Epstein (ed.), Capital Flight and Capital Controls in Developing Countries, Northampton (MA), Edward Elgar, 2005.

Cerra, V., M. Rishi and S.C. Safena, Robbing the Riches: Capital Flight, Institutions and Instability, IMF Working Paper, Nr. 199, Washington, DC, 2005, <http://www.imf.org>.

Christian Aid, Enough is Enough: The Debt Repudiation Option, London, 2007, <http://www.christianaid. org $>$.

Concord and Eurodad, Hold the Applause! EU Governments Risk Breaking Aid Promises, Brüssel, April 2007, <http://www.concordeurope.org > und <http://www.eurodad.org >.

Eurodad (ed.), EU Aid: Genuine Leadership or Misleading Figures? An Independent Analysis of European Aid Figures, Joint European NGO Report, April 2006, <http://www.concordeurope.org> und $<$ http://www.eurodad.org $>$.

Eurodad, Skeletons in the Cupboard: Illegitime Debt Claims of the G7, Eurodad, 2007.

Henry, James S., „The Mirage of Debt Relief“, in Steven Hiatt (ed.), A Game as Old as Empire: The Secret World of Economic Hit Men and the Web of Global Corruption, San Francisco, BerrettKoehler, 2007.

Hersel, Philipp und Sebastian von Eichborn, Investoren als Entwicklungshelfer? Zur Rolle von Krediten, Direktinvestitionen und Portfolio-Anlagen in Entwicklungsländern, Berlin, Berliner Landesarbeitsgemeinschaft Umwelt und Entwicklung (BLUE 21); Bonn, Evangelischer Entwicklungsdienst, 2006, $<$ http://www.blue21.de> und <http://www.eed.de>

International Monetary Front (IMF), World Economic Outlook, verschiedene Nummern, <http://www. imf.org/weo>.

Martens, Jens, Die öffentliche Armut der Entwicklungsländer, 2006, <http://www.nordsuednetz.de>.

Ocampo, José Antonio, Jan Kregel und Stephany Griffith-Jones (Eds.), International Finance and Development, London, Zed Books, 2007.

Oxfam, Tax Havens: Releasing the Hidden Billions for Poverty Eradication, Oxfam Briefing Papers, Oxford, 2000, <http://www.oxfam.org.uk>.

Schneider, Benu, Measuring Capital Flight: Estimates and Interpretation, ODI Working Paper, Nr. 194, London, Overseas Development Institute (ODI), März 2003, <http://www.odi.org.uk>.

Singh, Kavaljit, Why Investment Matters: The Political Economy of International Investments, London; Brüssel, FERN; Sturminster Newton (UK), The Corner House; Rom, Campagna per la riforma della Banca Mondiale (CRBM); Delhi, Madhyam Books, 2007, <http://www.fern.org> und <http://www. thecornerhouse.org.uk>.

Smith, Patrick, „On the Wing: How Capital Flight Is Cheating Africa“, The Africa Report, Nr. 2, März 2006, <http://www.capitalismsachillesheel.com/AfricaReportArticle.pdf>.

Tax Justice Network (TJN), Closing the Floodgates: Collecting Tax to Pay for Development, Paper commissioned by the Norwegian Ministry of Foreign Affairs, London, 2007, <http://www.taxjustice.net>.

United Nations, World Economic Situation and Prospects 2007, New York, 2007, <http://www.un.org/ esa/policy/wess/wesp> und <http://www.unctad.org $>$.

World Bank, Global Development Finance 2006, Vol. 1 and 2, Washington, DC, The World Bank, 2006, $<$ http://www.worldbank.org/gdf2006>.

\section{Internet-Adressen}

Internationaler Währungfonds (IWF) : <http://www.imf.org >

OECD-Entwicklungshilfeausschuss (DAC) : <http://www.oecd.org/dac $>$.

Pariser Klub : <http://www.clubdeparis.org $>$.

UNCTAD : <http://www.unctad.org >.

Weltbank: <http://www.worldbank.org $>$. 